\title{
Influence of bone microstructure distribution on developed mechanical energy for bone remodeling using a statistical reconstruction method
}

Mathematics and Mechanics of Solids $1-15$

(C) The Author(s) 2019 Article reuse guidelines: sagepub.com/journals-permissions DOI: $10.1177 / 1081286519828418$ journals.sagepub.com/home/mms (SAGE

\section{Azadeh Sheidaei}

Aerospace Engineering Department, lowa State University, Ames, IA, USA

\section{Morteza Kazempour}

School of Mechanical Engineering, College of Engineering, University of Tehran, Tehran, Iran

\section{Ali Hasanabadi}

Mechanical Engineering Department, University of Birjand, Birjand, Iran

\section{Fayyaz Nosouhi}

School of Mechanical Engineering, College of Engineering, University of Tehran, Tehran, Iran

\section{Martine Pithioux}

University of Aix-Marseille, APHM, CNRS, ISM, and Sainte-Marguerite Hospital, Institute for Locomotion, Marseille, France

\section{Majid Baniassadi}

School of Mechanical Engineering, College of Engineering, University of Tehran, Tehran, Iran

\section{Yves Rémond}

University of Strasbourg, CNRS, ICUBE Laboratory, Strasbourg, France

\section{Daniel George}

University of Strasbourg, CNRS, ICUBE Laboratory, Strasbourg, France

Received 30 October 2018; accepted 12 January 2019

Corresponding author:

Daniel George, University of Strasbourg, CNRS, ICUBE Laboratory, Strasbourg, France.

Email: george@unistra.fr 


\begin{abstract}
The development of a predictive model for bone remodeling is becoming increasingly important for medical applications such as bone surgery or bone substitutes like prostheses. However, as bone remodeling is a complex multiphysics phenomenon and difficult to quantify experimentally, predictive numerical models remain, at best, phenomenologically driven. Patient dependency is often ignored as its influence is usually considered secondary, although it is known to play an important role over long periods of time. Another difficulty to study this patient dependency is the availability of experimental samples to carry out extensive analyses. Using our recently developed statistical reconstruction framework, a set of "bone like" microstructures with variety of distributions has been created to study pseudo "patient variabilities." The method provides similar effective stiffness tensor, equivalent stresses, and strain energy distributions for the original and the statistically reconstructed samples. The main outcome of this study is the correlation of similar effective mechanical properties between samples when bone remodeling will depend on the local strain energy distribution as a function of each bone microstructure. It is expected that two different microstructures with equivalent bone volume fraction will lead to identical bone remodeling in a short period of time, whereas this needs to be proven for long term evolution. This work could be used to develop precise predictive numerical models while developing parametric studies on an infinite number of virtual samples and correlating patient dependency with more precise mechanobiological numerical models.
\end{abstract}

\title{
Keywords
}

Bone microstructure, statistical reconstruction, finite element model, mechanical behavior

\section{Introduction}

It is well established in the literature that the interaction between mechanics and biology plays a crucial role in the interpretation of growing tissue behavior [1-3]. This mechanobiology depends on the natural tendency of the biological tissue to develop optimizing behavior to gain proper mechanical strength with the minimum amount of material. For bone adaptation and remodeling, this optimization problem is at the origin of many studies since the early work by Wolff [4] followed by many others [5-9] exploring the internal architecture and external conformation of bones changes in accordance with mathematical laws. The evolution of bone microstructure being dependent on cells and microstructure distribution, various mechanical stimuli have been proposed to trigger bone adaptation [10-13]. While continuum models, for the description of natural bone regeneration, are widely spread in the scientific literature, scale related effects of the material, are not often integrated in the structural evolution due to its highly heterogeneous nature at the small scales. At these scales, the classical continuum theory does not allow to describe the correct material behavior where the prediction made by Cauchy continuum theory starts being far away from the experimental evidence [14-18]. Hence, adequate homogenization methods [19,20] need to be developed in order to integrate all these local phenomena at the global scale.

Historically, bone mechanical behavior was dealt with using classical optimization processes based mainly on the developed mechanical strain energy density (SED) through the material [21-29]. However, to be able to obtain a good predictive numerical model, the biological phenomena [30] need to be linked with the surrounding physics [31-35], and account for the growing capillaries and nutrient supply [36-39]. Lately, a new form of mechanobiological stimulus for bone remodeling has emerged integrating coupled biological and mechanical effects accounting for cell migration and the nutrient supply chain [40-42]. Although this allows accounting for the couplings between the mechanics and biological phenomena, it remains a macroscopic approach and requires more insight about the locally occurring mechanobiology.

Bone microstructure distribution is optimized in order to sustain the externally applied mechanical loads and its evolution changes over the long term as a function of local biological information. However, on a macroscopic scale, where the bone density is characterized only as a homogeneous media, the influence of this microstructure over the long term remodeling kinematics is little investigated. Although some numerical models tried to predict the bone remodeling kinematics from known trabecular bone distributions [43-46], these do not provide insight about long term evolutions. In addition, patient dependent bone microstructure is very variable [47,48], this could impact directly the numerical model predictions. Lately, some numerical models have investigated the correlations between the bone stiffness and simplified microstructure distribution [49-53] through multiscale homogenization procedures. However, the bone remodeling processes accounting for these microstructure variabilities at 
the macroscopic scale are not studied since these works are mostly related to the structural mechanics homogenization process.

The ultimate goal of this research is to study the influence of the local bone microstructure distribution on the macroscopic bone density evolution over long periods of time. The first step of this study require an adequate method for bone microstructure reconstruction in order to study large sets of specimens as it is not possible to extract that amount of real bone samples, and show that the proposed reconstruction method is able to provide corresponding results with different structural samples as equivalent effective stresses, strain energy and stiffness tensor. For this, we present here a methodology to study the influence of the bone microstructure distribution on the developed mechanical energy field. Real bone microstructures are extracted from computed tomography (CT) scan data and segmented/reconstructed to obtain the corresponding finite element (FE) numerical model. A statistical method is used to reconstruct several equivalent bone microstructures with identical homogeneous stiffness. FE models are then constructed from these statistical microstructures. The same mechanical loads are applied on the different FE models to extract the variabilities of the local SED that will be at the origin of the bone remodeling kinetics.

\section{Experimental details}

\section{I. Specimen preparation}

The real bone specimen was extracted from the femur of an 85 -year-old woman post mortem. The adult bone was harvested from the corpse at the Timone Hospital (Marseille, France). The specimen was cut into a parallelepiped rectangle of length, width, and thickness dimensions $9.88 \mathrm{~mm}, 6.13 \mathrm{~mm}$, and 2.29 $\mathrm{mm}$, respectively, using a water-cooled low-speed diamond saw (Buehler Isomet 4000, Buehler, Lake Bluff, IL, USA). The length of each specimen was measured with a digital calliper (Absolute digimatik solar, Mitutoyo, Kanagawa, Japan, measurement error of $0.03 \mathrm{~mm}$ ). The specimen was stored under vacuum at $-20^{\circ} \mathrm{C}$.

\subsection{Image acquisition}

The specimen was imaged at $5.5 \mu \mathrm{m}$ voxel size using a micro-CT system (Phoenix Nanotom $180 \mathrm{~S}$, General Electric, Germany). The scanning was done with a hydrated bone specimen, immersed in salt water inside a $25 \mathrm{~mm}$ inner diameter plastic tube. The axial specimen main axis (i.e., Haversian canal's principal orientation and medullar canal orientation) was aligned with the rotational sample holder axis. Scans were done with a field of view of $2284 \times 2304$ pixels, with a maximum voltage source of 90 $\mathrm{kV}$, a current of $120 \mu \mathrm{A}$ and a rotation of $360^{\circ}$ providing 2400 projections per scan. The exposure time was $500 \mathrm{~ms}$ with a total scan time of 85 minutes. The bone density was measured at $92 \%$ of the overall specimen volume.

\section{Theoretical modeling}

The statistical reconstruction approach enables to obtain various different microstructures with identical effective stiffness tensor by exploiting a single cut section image through the original bone sample. Bone images can be extracted from CT-scan or other experimental techniques.

Following our previous works [54,55], several bone microstructures were generated with identical effective stiffness tensor to evaluate the effect of the geometrical distribution of bone microstructure on local energy. This enabled to study many different scenarios (different virtual samples), using the full spectrum of two-point correlation function (TPCF) for eigen microstructure, without the need of extracting real bone samples.

The characteristic function $(\mathrm{CF})$ of the heterogeneous microstructure can be written [56] as:

$$
\kappa_{s}^{n}= \begin{cases}1 & \text { if } \mathrm{s} \in \text { phase } n \\ 0 & \text { otherwise }\end{cases}
$$

where $n$ is a specific phase and the $s$ index enumerates the voxel number of the sample. 
This method is based on the fact that the material is not a functionally graded material (FGM) and that the statistical information of parallel cut sections are identical.

To reconstruct a three-dimensional (3D) representative volume element (RVE) based on given twodimensional cut-sections, we introduce TPCFs, being statistical functions that give some information about the volume fraction, shape and distribution of the microstructure phases and are correlated with its various features such as effective thermal and mechanical properties [56].

In this research, we use fast Fourier transform (FFT) and a phase recovery algorithm to reconstruct the microstructure [57]. The TPCF of the phases $n$ and $n^{\prime}, C_{2}^{n n}$, for an arbitrary vector $\boldsymbol{t}$, can be defined as

$$
C_{2}^{n n^{\prime}}(\boldsymbol{t})=\frac{1}{S} \sum_{s=1}^{S} \kappa_{s}^{n} \kappa_{s+\boldsymbol{t}}^{n^{\prime}}
$$

where $S$ is the number of all voxels in the RVE and $\boldsymbol{t}$ is an arbitrary vector selected from the RVE. The Fourier transform (FT) of equation (2) leads to a more convenient form [58] for computation:

$$
\widehat{K}_{\boldsymbol{m}}^{n n^{\prime}}=\mathcal{F}\left(C_{\boldsymbol{s}}^{n n^{\prime}}\right)=\frac{1}{S} \sum_{\boldsymbol{t}=1}^{S} \sum_{\boldsymbol{s}=1}^{S} \kappa_{\boldsymbol{s}}^{n} \kappa_{\boldsymbol{s}+\boldsymbol{t}}^{n^{\prime}} \mathrm{e}^{-2 \pi \mathrm{i}(\boldsymbol{t} \cdot \boldsymbol{m}) / S}
$$

Using the variable exchange $(\boldsymbol{t}=\boldsymbol{z}-\boldsymbol{s})$ and assuming a periodic extension of the RVE in all directions, equation (3) can be written as

$$
\widehat{K}_{\boldsymbol{m}}^{n n^{\prime}}=\mathcal{F}\left(C_{\boldsymbol{s}}^{n n^{\prime}}\right)=\frac{1}{S} \sum_{\boldsymbol{s}=1}^{S} \boldsymbol{\kappa}_{s}^{n} \mathrm{e}^{-2 \pi \mathrm{i} \cdot \boldsymbol{m} / S} \sum_{\boldsymbol{z}=1}^{S} \kappa_{s}^{n^{\prime}} \mathrm{e}^{2 \pi \mathrm{i} z \boldsymbol{m} / S}=\frac{1}{S}\left|\widehat{K}_{\boldsymbol{m}}^{n}\right| \mathrm{e}^{-\mathrm{i} \theta_{m}^{n}}\left|\widehat{K}_{\boldsymbol{m}}^{n^{\prime}}\right| \mathrm{e}^{\mathrm{i} \theta_{\boldsymbol{m}}^{n^{\prime}}}
$$

where $\left|\widehat{K}_{m}^{n}\right|$ and $\theta_{m}^{n}$ are the module and phase of the FT for the CF, with

$$
\widehat{K}_{\boldsymbol{m}}^{n}=\mathcal{F}\left(\kappa_{\boldsymbol{s}}^{n}\right)=\sum_{\boldsymbol{s}=1}^{S} \kappa_{\boldsymbol{s}}^{n} \mathrm{e}^{2 \pi \mathrm{i} \cdot \boldsymbol{m} / S}=\left|\widehat{K}_{\boldsymbol{m}}^{n}\right| \mathrm{e}^{\mathrm{i} \theta_{\boldsymbol{m}}^{n}}
$$

It should be noted that the FT, expressed as $\mathcal{F}($.$) , is calculated over all axes (i.e. 3D FT for \left(s_{1}, s_{2}, s_{3}\right)$ ).

In equation (3), if $n=n^{\prime}$ the TPCF is also called autocorrelation function, and its FT, using equation (4), can be obtained as

$$
\widehat{K}_{\boldsymbol{m}}^{n n}=\frac{1}{S}\left|\widehat{K}_{\boldsymbol{m}}^{n}\right|^{2}
$$

Equation (6) shows that the FT of the autocorrelation function is related only to the FT module of the $\mathrm{CF}$. If the FT of the autocorrelation functions exist, it is then possible to calculate its modules. Hence, for a two-phase microstructure, if the autocorrelation functions are to be known at first, it is possible to reconstruct the microstructure if the phase of the microstructure (i.e. $\theta_{m}^{n}$ ) is determined (see equation (5)). The reconstruction of an object based just on its FT modules, called phase recovery [59], is a common method in astronomy and microscopy.

The application of this procedure for microstructure reconstruction was first introduced by Fullwood et al. [58]. The reconstruction procedure using the phase recovery algorithm is as follow:

(1) Based on the known autocorrelation functions, the module of the CF is calculated using equation (6). It is called the original amplitude. From this first step a random microstructure is generated.

(2) Once the FT of the randomly generated microstructure calculated, its amplitude is replaced by the original amplitude and then the inverse FT is calculated.

(3) Constraints of the generated microstructure are applied. If the difference between the FT amplitude of the obtained $\mathrm{CF}$ and the original amplitude is below a given threshold, the reconstruction procedure is considered completed and acceptable. Else, the obtained CF is defined inadequate 




Figure I. Decomposition of the vector $\boldsymbol{r}$ to $\boldsymbol{r}_{z}$ and $\boldsymbol{r}_{x y}$.

for this generated microstructure and the procedure is reset until the difference between the two amplitudes falls under the given threshold.

An improvement of this method was recently proposed by Hasanabadi et al. [54,55] on a new approximation for the calculation of the TPCFs for a 3D RVE. We assume that the TPCF of an identical vector in a given RVE in two parallel planes are approximately equal. Hence, only two perpendicular cutsections (planes) in the RVE are necessary for the TPCFs definition and reconstruction process. This assumption is valid for statistically homogenous media [57]. As shown in Figure 1, an arbitrary vector $\boldsymbol{r}$ in space can be decomposed into $\boldsymbol{r}_{z}$ and $\boldsymbol{r}_{x y}$. If the TPCFs of two planes, $x y$ and $y z$ are known, the full set of TPCF for 3D RVE can be approximated [55] based on

$$
C_{2}^{11}(\boldsymbol{r}) \approx \frac{C_{2}^{11}\left(\boldsymbol{r}_{x y}\right) C_{2}^{11}\left(\boldsymbol{r}_{z}\right)}{v_{1}}+\frac{\left(v_{1}-C_{2}^{11}\left(\boldsymbol{r}_{x y}\right)\right)\left(v_{1}-C_{2}^{11}\left(\boldsymbol{r}_{z}\right)\right)}{\left(1-v_{1}\right)}
$$

where $v_{1}$ is the volume fraction of phase 1 .

The flowchart of the reconstruction procedure is presented in Figure 2. More details of this method can be found in [58].

In this approach, TPCFs were extracted using CT images and used to reconstruct microstructures converging to the real ones. The main reasons to choose this reconstruction technique are as follows:

(i) A strong contrast expansion shows that the stiffness tensor is linked to the TPCF of the microstructure; therefore the TPCF is a very good selection of the statistical distribution descriptor.

(ii) In the phase recovery algorithm, different sets of TPCF can be employed for the reconstruction procedure, therefore enabling to reconstruct a variety of microstructures with few cut-section images.

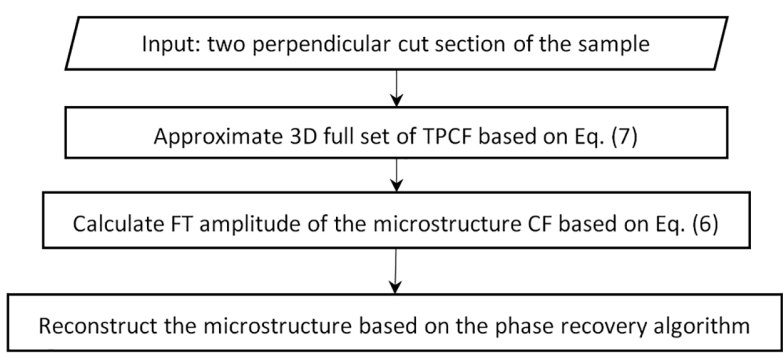

Figure 2. Flowchart of the reconstruction procedure. 


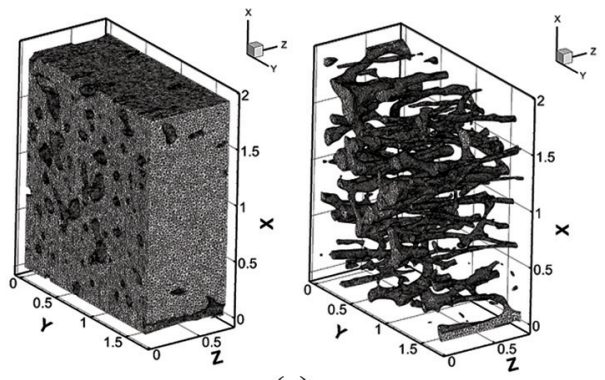

(a)

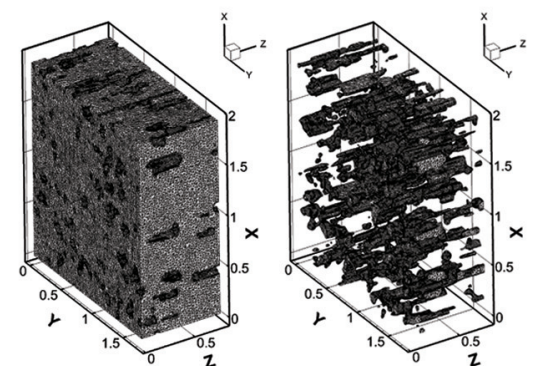

(b)

Figure 3. Finite element mesh for cortical bone microstructures (left: bone phase, right: void phase): (a) real specimen; (b) statistically reconstructed specimen from cut section 2 . The approximate size of samples (real and reconstructed ones) is $1930 \mathrm{x}$ $1750 \times 600 \mu \mathrm{m}^{3}$.

An improvement of the reconstruction process is envisaged in future works using two-point cluster functions but the mathematical framework for this needs to be developed.

\section{Finite element model}

The original CT scanned images were cropped into size $351 \times 319$ pixels and were imported into the VCAT software (released by V-CAD Program, RIKEN, Japan). The images were then assembled into stacks of 150 images after thresholding and made a 3D RVE of the bone microstructure. Figure 3 shows the 3D RVE for the real bone specimen and the statistical one possessing similar realistic features (size, shape, and distribution) as the real one, being suitable for the calculation of its mechanical properties. Figure 3 also shows the connected network of voids inside the cortical bone. Effective properties were extracted directly from the results of the FE analysis on statistical and real bone microstructures. A direct comparison was made between the real bone sample and statistical reconstructed ones to validate the reconstruction process.

In this work, the primary goal is to focus on the ability of the statistical reconstruction method to provide "bone like" microstructures and check whether these reconstructed microstructures show similar mechanical characteristics as the real bone. Once the statistical reconstruction method validated on a number of samples, it is then possible to create virtually an infinite number of microstructures showing different microstructures distributions and bone densities, hence enabling to study an infinite number of "bone samples" without the need of specimen extraction. Although the computation time is an important aspect, this is not the primary focus here as it can be addressed in future studies, but rather crucial to validate the quality of the reconstruction method in the first place.

The V-CAT software was used with its built-in mesh generator to obtain a high quality tetrahedral FE mesh, accounting for highly irregular and high aspect ratio solid and void clusters. This capability enabled to preserve a good quality of the void volume fraction during the meshing process. The mesh simplifier software has been used to reduce the number of elements in the bone part without sacrificing the volume fraction of the void. The total number of elements in the bone part was around 3.5 million tetrahedral elements for all RVEs. The volume fraction of the void phase has been calculated around $8 \%$, which is very close to the original CT scanned data. More details about creating the 3D model, mesh generation and homogenization process for real and reconstructed microstructures are provided in [60].

The real internal bone microstructure either cortical or trabecular is subjected to the combination of normal and shear loads defined by the external bone geometry and load conditions (muscles, body weight, bone type). However, it is rather difficult to extract quantitative data from such conditions. Hence, in order to be able to validate the statistical reconstruction method by comparing developed stresses and elastic strain energy on real and statistical bone microstructures, we conducted six simulations (three tensile tests and three shear tests) on each RVE using the FE software ABAQUS ${ }^{\circledR}$. These were used to find the modulus of elasticity and Poisson's ratios of the RVEs in the X, Y, and Z directions. 
Since bone is a highly heterogeneous material $[61,62]$ and this affects its overall mechanical behavior $[63,64]$, it is necessary to compare the obtained results at the effective macroscopic scale. Based on these tests, stiffness tensors of all RVEs were obtained and influences of the microstructure distributions were quantified.

\section{Results and discussion}

Five statistical bone microstructures were reconstructed (see example in Figure 3). From these different cases, including real bone sample, homogenized macroscopic stiffness coefficients (i.e. Young modulus and Poisson's ratio) were extracted for the validation of the reconstruction process, assuming the material (bone solid phase) mechanical behavior was linear elastic with a Young modulus $E=15 \mathrm{GPa}$ (average value for cortical bone) and Poisson's ratio $v=0.3$. The results are presented in Table 1 . The real bone sample presented values of Young modulus varying between $11.17 \mathrm{GPa}$ and $12.89 \mathrm{GPa}$, and Poisson's coefficients between 0.228 and 0.301 in the different directions. It is observed that the Young moduli $E_{x}$ and $E_{y}$ are the lowest and $E_{z}$ is the highest which is due to the fact that cortical bone microstructure is orientated along the Haversian canal. Comparing these values with the obtained homogenized coefficients of all statistically reconstructed cases, it was observed that for all of them, the obtained Young moduli are very close to the minimum value obtained for $E_{y}$ of section 6 at $10.76 \mathrm{GPa}$ and highest value for $E_{z}$ of sections 2 at $13.326 \mathrm{GPa}$. The errors developed from the microstructures variations for the Young moduli are; for the minimum Young modulus value equal to $3.1 \%$, for the maximum Young modulus value equal to $3.38 \%$ and for the range min-max equal to $4.39 \%$. For the Poisson's ratio, their variability checked between 0 and 0.06 , which is a very small range. In addition, it was observed that the real sample mechanical properties are mostly situated in the middle of all reconstructed cases which show a good correlation with the different scenarios.

From the original bone sample, it was possible to reconstruct different bone microstructures corresponding to different case scenario (different samples/different virtual patients) with a very good accuracy. The reconstruction method allows obtaining an infinite number of virtual cases (patients) from the real bone sample, to study their mechanical behavior individually, and compare them with the real bone sample. It is also possible to extract tendencies with given scenarios of specific microstructure distributions.

Once the statistical reconstruction of different microstructure was validated, both tensile and shear mechanical loads were applied on each of them to compare their mechanical behavior, as well as the stresses and energy/energy density distribution fields developed. Each model is constituted of about 3.5 million tetrahedral FE elements in the solid phase. The applied load corresponds to an average standing person body weight (about $750 \mathrm{~N}$ ) downscaled to the size of the sample surfaces. This leads to small deformation in the sample for the given mechanical properties; hence linear elastic analysis is adequate here. For the sake of lightness of the paper, we present only the results of the real bone sample with two other statistical microstructures built from cut section 3 and cut section 5 on the real bone sample respectively.

For the tensile test, the stresses are plotted in the corresponding loading direction $(x)$ and $(z)$, together with the corresponding SED developed within the microstructures. They are presented in Figures 4 and 5 , respectively. For the shear load scenario, stresses in the $(x y)$ direction together with SED are presented in Figure 6. Although the effective equivalent mechanical properties are similar for all cases (see Table

Table I. Modulus of elasticity and Poisson's ratio in different directions for the real and statistical bone microstructures.

\begin{tabular}{llllllllll}
\hline & $E_{x}(\mathrm{GPa})$ & $E_{y}(\mathrm{GPa})$ & $E_{z}(\mathrm{GPa})$ & $v_{x y}$ & $v_{x z}$ & $v_{y z}$ & $v_{y x}$ & $\nu_{\mathrm{zx}}$ & $\nu_{z y}$ \\
\hline Real bone & 11.723 & 11.172 & 12.894 & 0.228 & 0.263 & 0.262 & 0.275 & 0.289 & 0.301 \\
Cut section 2 & 12.506 & 12.064 & 13.326 & 0.291 & 0.272 & 0.274 & 0.281 & 0.29 & 0.301 \\
Cut section 3 & 11.456 & 11.310 & 12.686 & 0.281 & 0.260 & 0.264 & 0.277 & 0.288 & 0.298 \\
Cut section 4 & 11.456 & 11.310 & 12.713 & 0.281 & 0.260 & 0.264 & 0.277 & 0.289 & 0.297 \\
Cut section 5 & 11.556 & 11.229 & 12.838 & 0.288 & 0.259 & 0.263 & 0.279 & 0.288 & 0.297 \\
Cut section 6 & 11.185 & 10.765 & 12.370 & 0.282 & 0.259 & 0.258 & 0.271 & 0.287 & 0.298
\end{tabular}




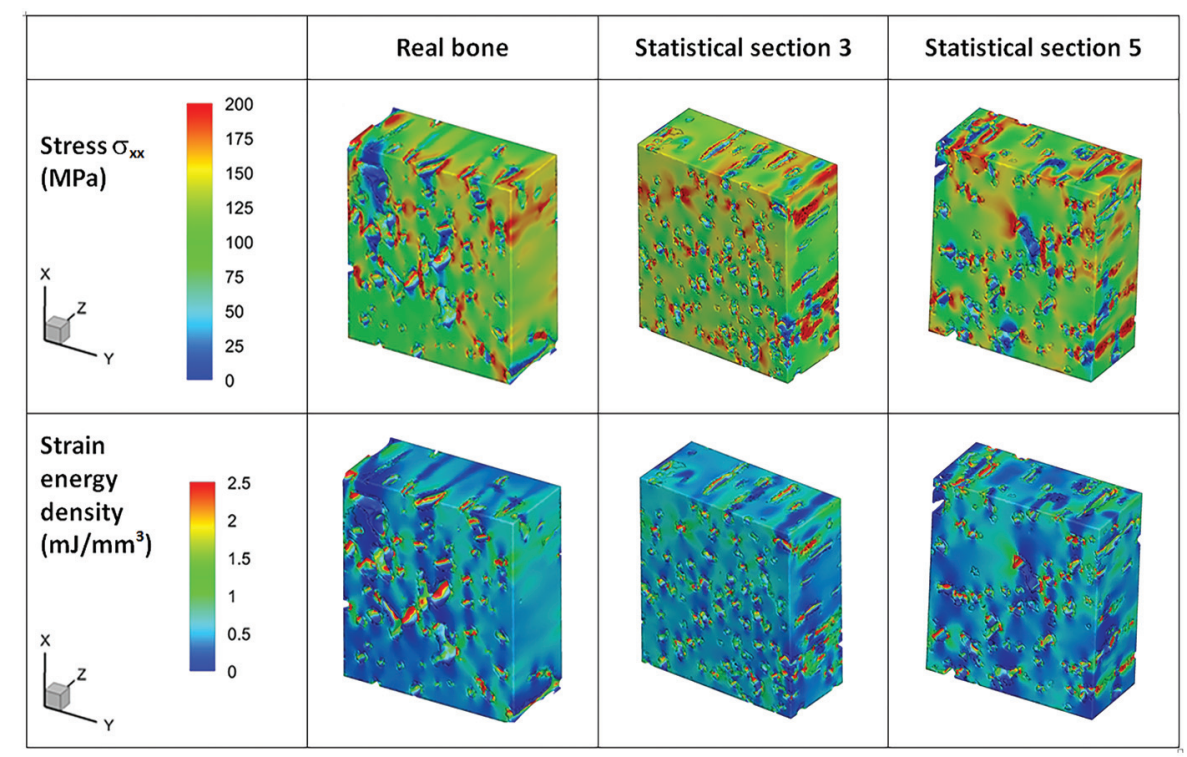

Figure 4. Developed stresses and strain energy density in the real bone and statistical samples (section 3 and 5 ) under tensile load applied in the $x$-direction.

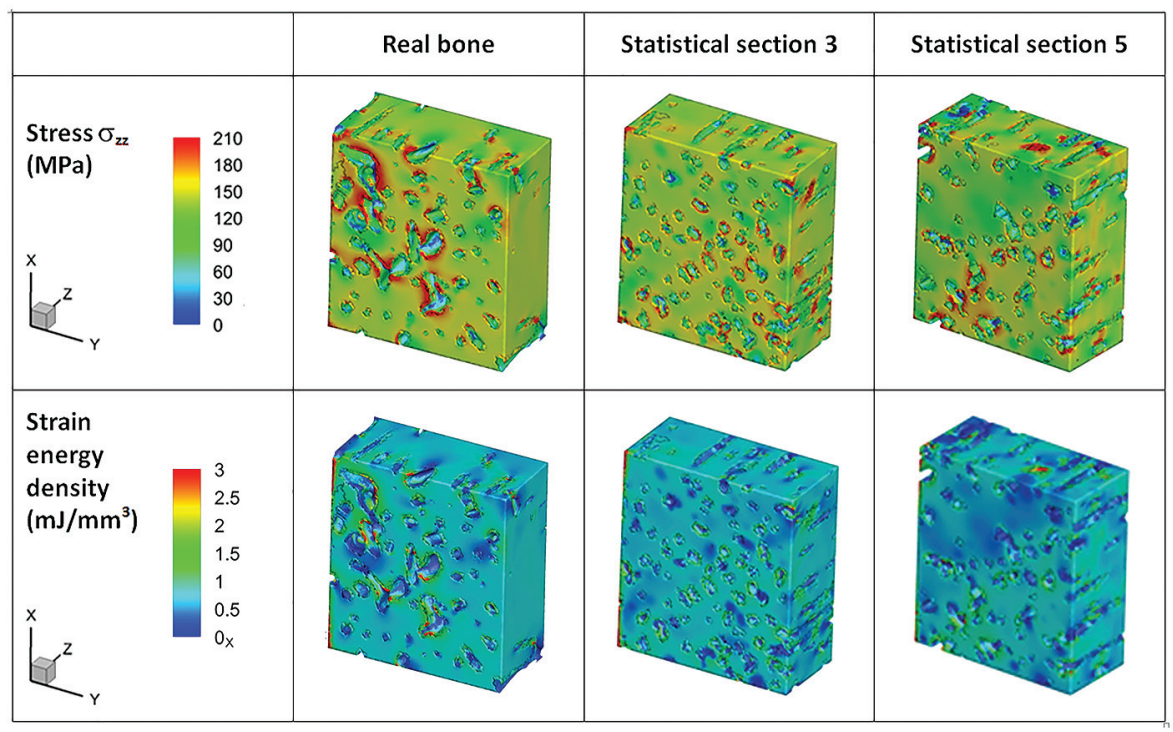

Figure 5. Developed stresses and strain energy density in the real bone and statistical samples (section 3 and 5 ) under tensile load applied in the $z$-direction.

1), the stresses and SED, presented in Figures 4 to 6, show different distributions for both tensile and shear applied loads. Despite this anisotropic distribution, the amount of developed energy within the samples remains similar (see below).

The interpretations of these results tend to show that:

(i) The statistical reconstruction method provides a practical method to study any virtual sample from the mechanical behavior point of view as homogenized mechanical variables (stiffness coefficients, stresses, and strain energy) remain very similar. It provides a viable method to extract local microstructures interpretations. More specifically, it shows the equivalent correlation 
between homogenized bone density measurements (as in magnetic resonance imaging information, for example, for the gray scale of the Hue value) and different types of microstructure distributions.

(ii) The variabilities of the mechanobiological response for each reconstructed case can be evaluated as a function of the distributed SED and the different microstructure distributions. This in turn can provide long term variations in the bone density evolution and can show the influence of each of the different microstructures on the bone density kinetics.

To evaluate the potential bone density evolution as a function of the applied mechanical loads, the total developed strain energy inside each sample for the different cases (real sample and five reconstructed cases) in tensile and shear load conditions are presented in Figure 7.

Small variations of the developed strain energy are observed for each of the different load scenarios. For tensile loads, the strain energy varies from a minimum of $1.485 \mathrm{~mJ} / \mathrm{mm}^{3}$ up to $1.786 \mathrm{~mJ} / \mathrm{mm}^{3}$. For shear loads, it varies from a minimum of $2.284 \mathrm{~mJ} / \mathrm{mm}^{3}$ up to $2.622 \mathrm{~mJ} / \mathrm{mm}^{3}$. For all cases, the real

\begin{tabular}{|c|c|c|c|c|}
\hline & & Real bone & Statistical section 3 & Statistical section 5 \\
\hline $\begin{array}{l}\text { Stress } \sigma_{x y} \\
\text { (MPa) }\end{array}$ & & & & \\
\hline$x$ & 60 & & & \\
\hline $\begin{array}{l}\text { Strain } \\
\text { energy } \\
\text { density } \\
\left(\mathrm{mJ} / \mathrm{mm}^{3}\right)\end{array}$ & $\begin{array}{l}2.5 \\
2 \\
1.5\end{array}$ & & & \\
\hline$x$ & 0.5 & & & \\
\hline$\theta^{2}$ & & & & \\
\hline
\end{tabular}

Figure 6. Developed stresses and strain energy density in the real bone and statistical samples (section 3 and 5 ) under shear load applied in the $x y$-direction.



Figure 7. Total developed strain energy for the different bone microstructures $(X, Y$, and $Z$ indicate tensile test and $X Y, X Z$, and YZ indicate shear tests). 
bone sample is always around the mid-value of the statistical cases in the same way as was observed for the stiffness coefficients. The strain energy variation for all cases is within 3\% to $6 \%$ compared to the real bone sample showing the reliability of the statistical reconstruction method. The developed strain energy in the $\mathrm{z}$ direction is slightly higher than in the $x$ and $y$ directions for tensile load conditions. This is in agreement with the higher stiffness of the $z$ direction reported in Table 1. However, interestingly, shear load cases show significantly higher strain energy than simple tensile load conditions. The average energy for shear load has increased by 53\% compared to tensile load conditions. This needs to be investigated in future work with a more exhaustive screening of different bone microstructure in order to extract the coupling effects between shear and tensile load conditions.

Nevertheless, as bone cells activation will depend on the developed mechanical energy within the structure, we can assume that for different types of bone microstructures, this coupling effect (between shear and tensile loads) will impact on the bone remodeling process. If shear stresses show to be predominant, it could be correlated to the fact that cells are more sensitive to shear load conditions than hydrostatic pressure [65], hence favoring shear loads for optimized bone reconstruction.

In order to correlate the viability of the reconstructed microstructures to the real bone sample, probability distribution functions (PDFs) were extracted. It represents the number of elements in the sample having a specific strain energy and is plotted as an element count versus strain energy divided by the total number of elements in the model. Figure 8 shows the average PDF value of the statistically reconstructed cases with the real bone PDF distribution. Statistical variations are small and a very good agreement is observed between the reconstructed cases mechanical response and the real sample.

Since mechanical load type, load direction and microstructure distribution impact directly the bone density evolution, the SED for all the elements in each RVE were extracted. The PDFs of the SED for each load case and microstructure are presented in Figure 9.

For each load case, the PDF of real bone is plotted in red. We observe that not only the overall mechanical response of the statistically reconstructed microstructures is similar to the one of real bone but also the distributions of strain energy densities are similar. The variabilities observed in these results are based on the fact that a 3D stochastic reconstruction technique has been used for reconstruction of the bone microstructure. The proposed method includes two steps: (i) a full set of TPCFs is approximated using correlation functions being extracted from two perpendicular images; (ii) the full set of approximated TPCFs are used to reconstruct microstructures using a phase recovery algorithm. Hence, the accuracy of the reconstructing technique can directly be related to the approximation of the full set of TPCF. In our previous study [66], it was showed that for anisotropic microstructure, the error of the estimated TPCF and consequently, the error of the reconstruction procedure increased in the direction with high anisotropic distribution of microstructure. In addition, the reference image used for the reconstruction is the source of errors as it depends on the approximated TPCF in the direction perpendicular to the microstructure distribution of the reference image [66].

Nevertheless, accounting for the anisotropic complexity of the trabecular bone microstructures, the differences obtained from the reconstruction method remain small, as presented in Table 2. Comparing these results with those in Figure 9 shows that:

(i) All strain energies over the whole sample for the statistical cases remain lower than $10 \%$ variations compared with the real bone sample. For simple tensile load cases, variations were between $0.2 \%$ and $4.5 \%$, showing the influence of the anisotropic microstructure distribution over the reconstruction method [66]. For shear load cases, the differences were up to $9.4 \%$ in the $y z$ direction showing in addition to the statistical error the sensitivity of the shear load scenario on the microstructure distribution, as presented on Figure 7.

(ii) For the standard deviation variations, they are not correlated to the strain energy variations as the mean strain energy does not account for the individual microstructure distributions. However, we can still observe that for tensile cases, the $z$ direction shows higher difference due to the stiffness difference with other directions and reconstruction method [66]. This does not appear in the shear load case as it is a tangential load. The full width at half maximum (FWHM) deviations of the PDF for tensile load remain within the $10 \%$ variability. For the $z$ direction, it is almost identical. 


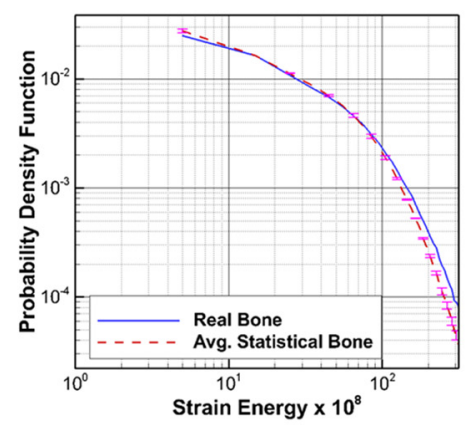

(a)

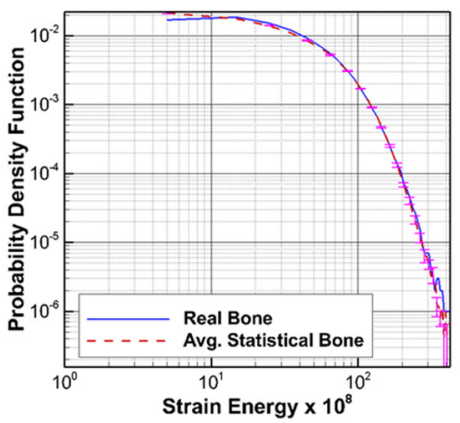

(b)

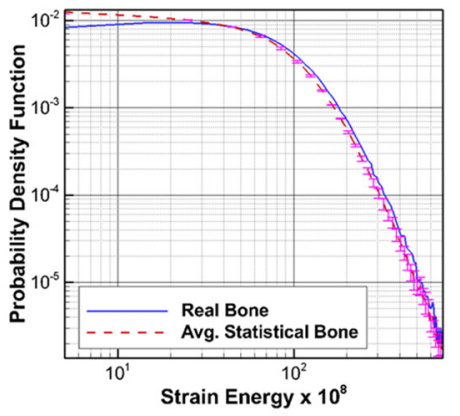

(c)

Figure 8. Probability density function for the $Y Y(a)$ and $Z Z$ (b) tensile cases, and $X Y$ (c) shear case as a function of the developed local strain energy $\left(\mathrm{mJ} / \mathrm{mm}^{3}\right)$.
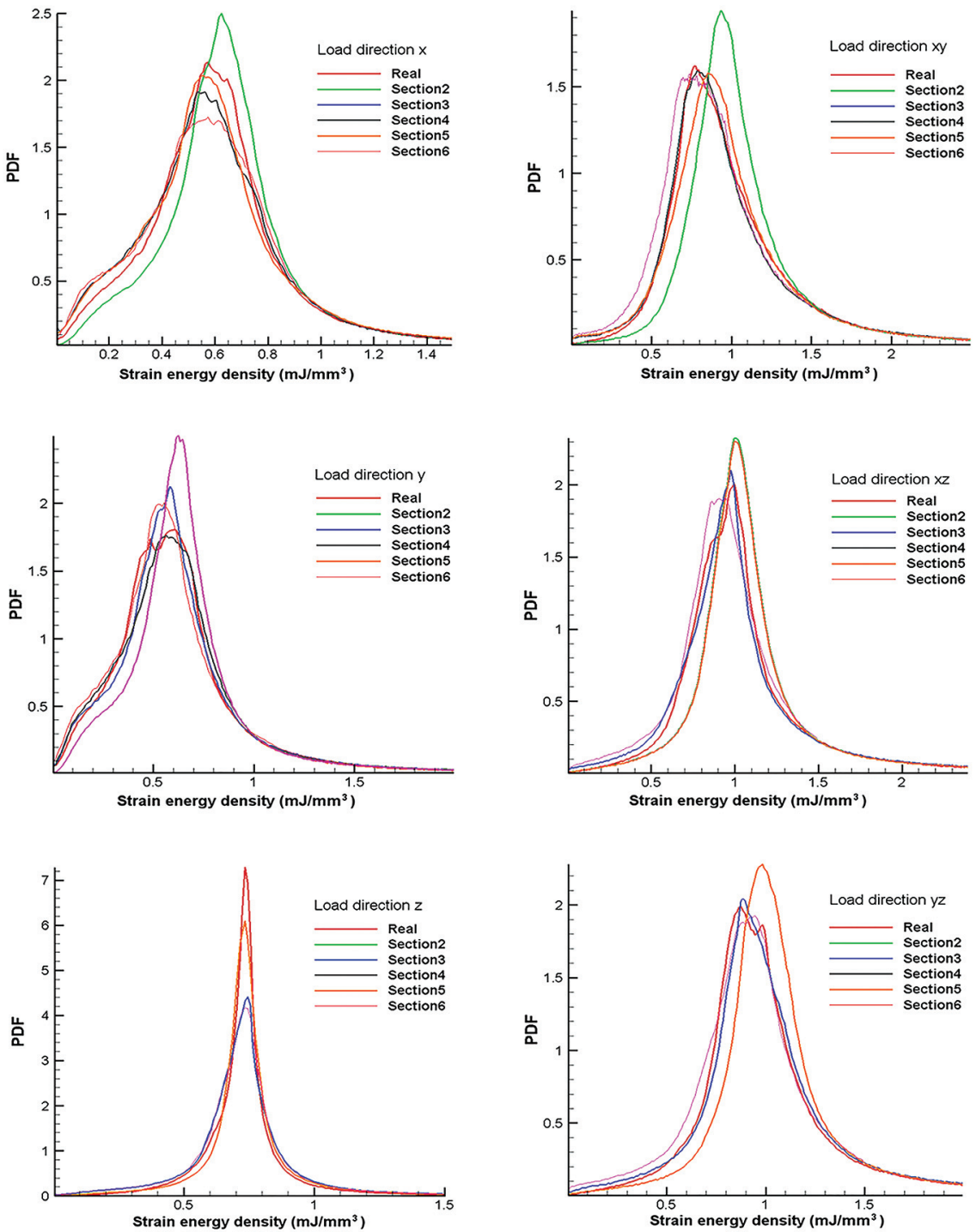

Figure 9. Normalized probability distribution function of strain energy density for the different microstructures with different mechanical load directions. 
It appears from the results in Table 1, Figures 7 to 9, and Table 2 that the statistical reconstruction method enables to study virtually an unlimited number of samples (bone like microstructures) as the calculated effective mechanical properties are very similar to the one of real bone. Some improvements remain to be done as the distribution of the strain energy shows variabilities over the statistical microstructures (up to $10 \%$ ) due to the abilities of the reconstruction method to best adapt to the real bone microstructure. These variations need to be evaluated with their respective impact on the bone density evolution over long periods of time in future works.

Table 2. Differences between real bone sample and averaged of statistical cases for: (i) strain energy density variation in different directions in tensile and shear load condition ( $X, Y$ and $Z$ indicate tensile test and $X Y, X Z$ and $Y Z$ indicate shear tests), and (ii) standard deviation difference at full width and half maximum (FWHM) as a representative of the variation of the energy density of each element compared to the mean value.

\begin{tabular}{lllllll}
\hline & Tension X & Tension Y & Tension Z & Shear XY & Shear XZ & Shear YZ \\
\hline $\begin{array}{l}\text { Mean strain energy } \\
\text { density variation }\left(\mathrm{mJ} / \mathrm{mm}^{3}\right)\end{array}$ & $0.0186(1.8 \%)$ & $0.0449(4.5 \%)$ & $0.0021(0.2 \%)$ & $0.0699(7 \%)$ & $0.0138(1.38 \%)$ & $0.0942(9.4 \%)$ \\
$\begin{array}{l}\text { Standard deviation } \\
\text { difference at FWHM }\end{array}$ & $11 \%$ & $10 \%$ & $42 \%$ & $6 \%$ & $11 \%$ & $7 \%$ \\
\hline
\end{tabular}

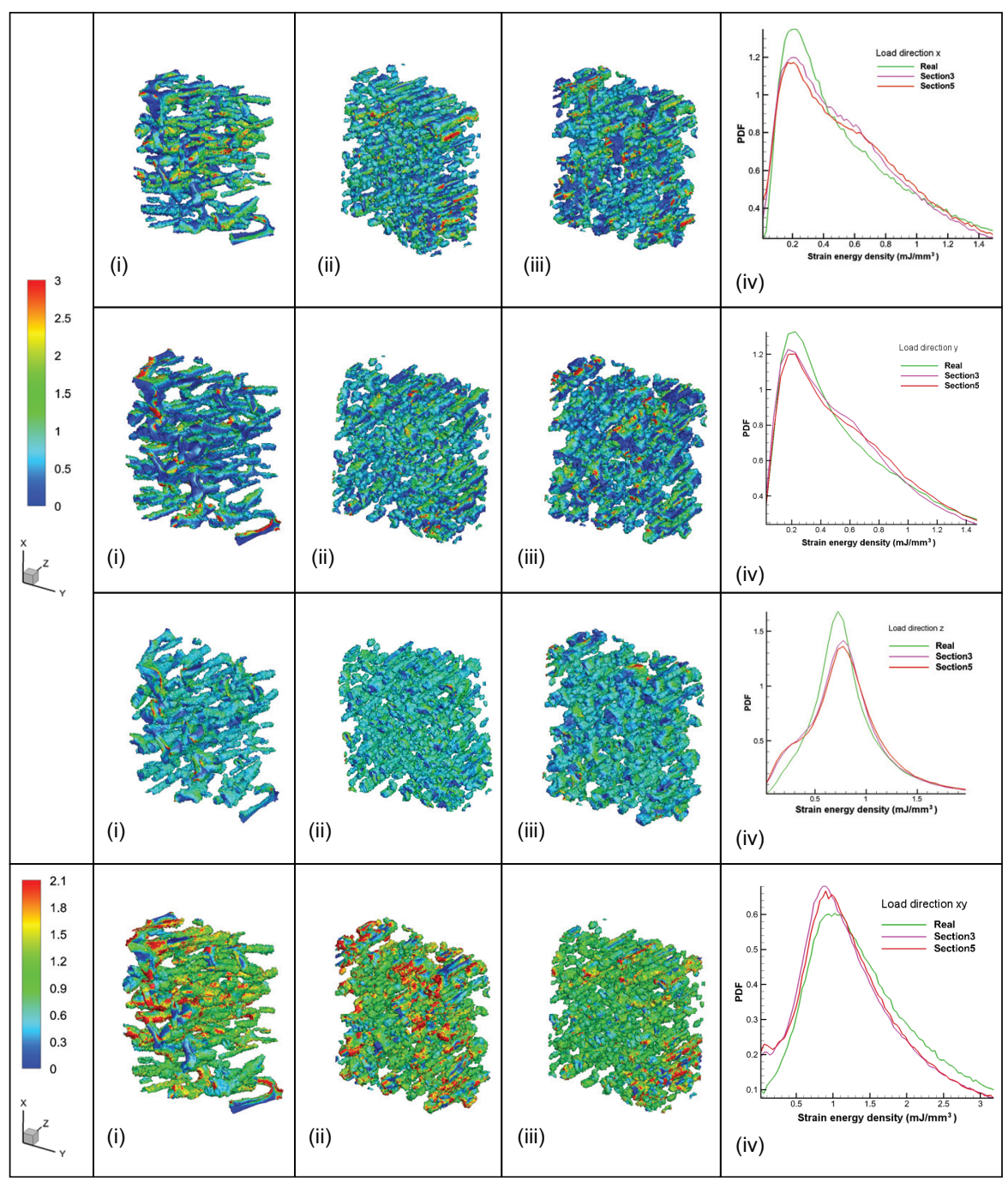

Figure 10. Strain energy density $\left(\mathrm{mJ} / \mathrm{mm}^{3}\right)$ on elements surrounding the void space for mechanical load applied in the $x, y, z$, and $x y$ directions: (i) real bone, (ii) cut section 3, (iii) cut section 5, and (iv) probability distribution function for the three cases. 
As bone remodeling occurs on internal surfaces of bone where osteoblasts and osteoclasts are present, it is necessary to look at the developed strain energy densities on these surfaces. These are presented on Figure 10 for three cases : (i) real bone, (ii) cut section 3, and (iii) cut section 5 for the mechanical load conditions of simple tension in $x, y$, and $z$ directions and shear $x y$. In addition, the PDF functions vs SED are plotted for the elements on the border. The tail of the PDF plots beyond a given energy is not shown on the figures as their PDF values are very small.

The presented results highlight the geometrical localization where bone remodeling is more likely to occur as osteoblasts and osteoclasts (respectively responsible of construction and resorption) are located on the bone surface. Similar to previous results, the maximum distributed SED is for shear load scenario with an average value around $1 \mathrm{~mJ} / \mathrm{mm}^{3}$ whereas for tensile loads, the average values are around $0.2 \mathrm{~mJ} /$ $\mathrm{mm}^{3}$ for $x$ and $y$ tensile loads and $0.7 \mathrm{~mJ} / \mathrm{mm}^{3}$ for $z$ tensile load. It appears again that tensile loads provide similar results compared to the stiffness coefficients proportions. We also observe that the real bone sample shows mostly higher SED for tensile loads but lower for shear load. The statistical samples are very close to each other, which could be correlated with the reconstruction method, as explained above [66].

Nevertheless, as far as the mechanical properties are concerned, a very good correlation is observed between the statistical cases and real bone which therefore provides good confidence in the statistical distribution of the developed strain energy. The impact of the observed differences needs to be quantified with regard to the biological distribution of cells on the developed surface microstructures and cell activation thresholds for bone remodeling. This will be done in future works.

\section{Conclusion}

A statistical reconstruction method was utilized to reconstruct cortical bone-like microstructures. The method is based on TPCFs using two perpendicular cutting planes within a real bone sample. Unlimited statistical bone microstructure distributions can be obtained with this method enabling the study of the influence of bone microstructure distribution without the need of extracting many patient bone samples.

All statistically reconstructed samples had identical effective bone density compared to the real bone sample and showed similar mechanical properties. The local stress, strain, and strain energy were calculated for all samples under simple mechanical load conditions. Local variations were observed as a function of the different reconstructed microstructures, and linked to effective values. Good correlation was observed for all cases.

The statistical reconstruction method enables us to create virtual bones, extract the variations of the local strain energy distribution as a function of the bone microstructure, and compare with macroscopic one. From this, bone remodeling could be incurred as a function of the local cell activation processes on the bone inner free surface. This local bone remodeling could therefore be linked to the effective bone density. The knowledge of the anisotropic stiffness tensor could then be used as a complementary indicator of the long term evolution of bone density. This needs to be validated in future work.

\section{Funding}

This research received no specific grant from any funding agency in the public, commercial, or not-for-profit sectors.

\section{References}

[1] Carter, DR, Van der Meulen, MCH, and Beaupré, GS. Mechanical factors in bone growth and development. Bone 1996; 18: S5-S10.

[2] Casanova, R, Moukoko, D, Pithioux, M, et al. Temporal evolution of skeletal regenerated tissue: what can mechanical investigation add to biological? Med Biol Eng Comput 2010; 48: 811-819.

[3] Huiskes, R, Ruimerman, R, van Lenthe, GH, et al. Effects of mechanical forces on maintenance and adaptation of form in trabecular bone. Nature 2000; 405: 704-706.

[4] Wolff, J. Das Gesetz der Transformation der Knochen. Berlin: Verlag von August Hirschwald, 1892.

[5] Cowin, SC. Wolff's law of trabecular architecture at remodeling equilibrium. J Biomed Eng 1986; 108: 83-88.

[6] Frost, HM. Bone "mass" and the "mechanostat": a proposal. J Anat Rec 1987; 219: 1-9.

[7] Lekszycki, T. Modeling of bone adaptation based on an optimal response hypothesis. Meccanica 2002; 37: 343-354. 
[8] Lekszycki, T. Functional adaptation of bone as an optimal control problem. J Theor Appl Mech 2005; 43: 120-140.

[9] Pivonka, P, Zimak, J, Smith, DW, et al. Model structure and control of bone remodeling: a theoretical study. Bone 2008; 43: 249-263.

[10] Hegedus, DH, and Cowin, SC. Bone remodeling II: small strain adaptive elasticity. J Elast 1976; 6: 337-352.

[11] Andreau, s U, Colloca, M, Iacoviello, D, et al. Optimal-tuning PID control of adaptive materials for structural efficiency. Struct Multidiscipl Optim 2011; 43: 43-59.

[12] Prendergast, PJ, and Taylor, D. Prediction of bone adaptation using damage accumulation. J Biomech 1994; 27: 1067-1076.

[13] Doblaré, M, and García, JM. Anisotropic bone remodelling model based on a continuum damage-repair theory. $J$ Biomech 2002; 35: 1-17.

[14] Dell'Isola, F, Andreaus, U, and Placidi, L. At the origins and in the vanguard of peridynamics, non-local and highergradient continuum mechanics: an underestimated and still topical contribution of Gabrio Piola. Math Mech Solids 2015; 20: 887-928.

[15] Misra, A, and Poorsolhjouy, P. Identification of higher-order elastic constants for grain assemblies based upon granular micromechanics. Math Mech Complex Syst 2015; 3: 285-308.

[16] Placidi, L, Andreaus, U, Della Corte, A, et al. Gedanken experiments for the determination of two-dimensional linear second gradient elasticity coefficients. Z Angew Math Phys 2015; 66: 3699-3725.

[17] Abali, BE, Müller, WH, and Dell'Isola, F. Theory and computation of higher gradient elasticity theories based on action principles. Arch Appl Mech 2017; 87: 1495-1510.

[18] Dell'Isola, F, Della Corte, A, and Giorgio, I. Higher gradient continua: the legacy of Piola, Mindlin, Sedov and Toupin and some future research perspectives. Math Mech Solids 2017; 22: 852-872.

[19] Rémond, Y, Ahzi, S, Baniassadi, M, et al. Applied RVE reconstruction and homogenization of heterogeneous materials. Wiley-ISTE, 2016.

[20] Martin, M, Lemaire, T, Haiat, G, et al. A thermodynamically consistent model of bone rotary remodeling: a $2 \mathrm{D}$ study. Comput Methods Biomech Biomed Eng 2017; 20(S1): 127-128.

[21] Madeo, A, Lekszycki, T, and Dell'Isola, F, A continuum model for the bio-mechanical interactions between living tissue and bio-resorbable graft after bone reconstructive surgery. C R Mécanique 2011; 339: 625-640.

[22] Lekszycki, T, and Dell'Isola, F. A mixture model with evolving mass densities for describing synthesis and resorption phenomena in bones reconstructed with bio-resorbable materials. Z Angew Math Mech 2012; 92: 426-444.

[23] Madeo, A, George, D, Lekszycki, T, et al. A second gradient continuum model accounting for some effects of microstructure on reconstructed bone remodeling. C R Mécanique 2012; 340: 575-589.

[24] Andreaus, U, Giorgio, I, and Lekszycki, T. A 2-D continuum model of a mixture of bone tissue and bio-resorbable material for simulating mass density redistribution under load slowly variable in time. Z Angew Math Mech 2014; 94: 978-1000.

[25] Scala, I, Spingarn, C, Rémond, Y, et al. Mechanically-driven bone remodeling simulation: application to LIPUS treated rat calvarial defects. Math Mech Solids 2016; 22: 1976-1988.

[26] Giorgio, I, Andreaus, U, Scerrato, D, et al. A visco-poroelastic model of functional adaptation in bones reconstructed with bio-resorbable materials. Biomech Model Mechanobiol 2016; 15: 1325-1343.

[27] Spingarn, C, Wagner, D, Rémond, Y, et al. Multiphysics of bone remodeling: a 2D mesoscale activation simulation. BioMed Mater Eng 2017; 28(s1): S153-S158.

[28] Cuomo, M. Forms of the dissipation function for a class of viscoplastic models. Math Mech Complex Syst 2017; 5: 217-237.

[29] Giorgio, I, Andreaus, U, Dell'Isola, F, et al. Viscous second gradient porous materials for bones reconstructed with bioresorbable grafts. Extreme Mech Lett 2017; 13: 141-147.

[30] Burr, DB, and Allen, MR. Basic and applied bone biology. Academic Press, 2013, 85-86.

[31] Pivonka, P, and Komarova, SV. Mathematical modeling in bone biology: From intracellular signaling to tissue mechanics. Bone 2010; 47: 181-189.

[32] Lemaire, T, Naili, S, and Sansalone, V. Multiphysical modelling of fluid transport through osto-articular media. An Ac Bras Ciências 2010; 82: 127-144.

[33] Lemaire, T, Capiez-Lernout, E, Kaiser, J, et al. What is the importance of multiphysical phenomena in bone remodelling signals expression? A multiscale perspective. J Mech Behav Biomed Mater 2011; 4: 909-920.

[34] Lemaire, T, Kaiser, J, Naili, S, et al. Three-scale multiphysics modeling of transport phenomena within cortical bone. Math Prob Eng 2015; 2015: 398970.

[35] Sansalone, V, Gagliardi, D, Descelier, C, et al. On the uncertainty propagation in multiscale modeling of cortical bone elasticity, Comput Methods Biomech Biomed Eng 2015; 18: 2054-2055.

[36] Bednarczyk, E, and Lekszycki, E A novel mathematical model for growth of capillaries and nutrient supply with application to prediction of osteophyte onset. Z Angew Math Phys 2016; 67: 94.

[37] Lu, Y, and Lekszycki, T. A novel coupled system of non-local integro-differential equations modelling Young's modulus evolution, nutrients' supply and consumption during bone fracture healing. Z Angew Math Phys 2016; 67: 111. 
[38] Moya, A, Paquet, J, Deschepper, M, et al. Human mesenchymal stem cell failure to adapt to glucose shortage and rapidly use intracellular energy reserves through glycolysis explains poor cell survival after implantation. Stem Cells 2018; 36: 363-376.

[39] Paquet, J, Deschepper, M, Moya, A, et al. Oxygen tension regulates human mesenchymal stem cell paracrine functions. Stem Cells Transl Med 2015; 4: 809-821.

[40] George, D, Allena, R, and Rémond, Y. Mechanobiological stimuli for bone remodeling: mechanical energy, cell nutriments and mobility. Comput Methods Biomech Biomed Eng 2017; 20: S91-S92.

[41] George, D, Allena, R, and Rémond, Y. A multiphysics stimulus for continuum mechanics bone remodeling. Math Mech Complex Syst 2018; 6: 307-319.

[42] Allena, R, and Maini, PK. Reaction-diffusion finite element model of lateral line primordium migration to explore cell leadership. Bull Math Biol 2014; 76: 3028-3050.

[43] Hollister, SJ, Brennan, JM, and Kikuchi, N. A homogenization sampling procedure for calculating trabecular bone effective stiffness and tissue level stress. J Biomech 1994; 27: 433-444.

[44] Tsubota, KI, Suzuki, Y, Yamada, T, et al. Computer simulation of trabecular remodeling in human proximal femur using large-scale voxel FE models: approach to understanding Wolff's law. J Biomech 2009; 42: 1088-1094.

[45] Jang, IG, and Kim, IY. Computational simulation of simultaneous cortical and trabecular bone change in human proximal femur during bone remodeling. $J$ Biomech 2010; 43: 294-301.

[46] Marzaban, A, Nayeb-Hashemi, H, and Vaziri, A. Numerical simulation of load-induced bone structural remodeling using stress-limit criterion. Comput Methods Biomech Biomed Eng 2013; 18: 259-268.

[47] Kersch, ME, Zysset, PK, Pahr, DH, et al. Measurement of structural anisotropy in femoral trabecular bone using clinicalresolution CT images. J Biomech 2013; 46: 2659-2666.

[48] Lian, WD, Legrain, G, and Cartraud, P. Image-based computational homogenization and localization: comparison between X-FEM/levelset and voxel-based approaches. Comput Mech 2013; 51: 279-293.

[49] Goda, I, Assidi, M, Belouettar, S, et al. A micropolar anisotropic constitutive model of cancellous bone from discrete homogenization. J Mech Behav Biomed Mater 2012; 16: 87-108.

[50] Rath, C, Baum, T, Monetti, R, et al. Scaling relations between trabecular bone volume fraction and microstructure at different skeletal sites. Bone 2013; 57: 377-383.

[51] Goda, I, Assidi, M, and Ganghoffer, JF. A 3D elastic micropolar model of vertebral trabecular bone from lattice homogenization of the bone microstructure. Biomech Mod Mech 2014; 13: 53-83.

[52] Wierszycki, M, Szajek, K, Lodygowski, T, et al. A two-scale approach for trabecular bone microstructure modeling based on computational homogenization procedure. Comput Mech 2014; 54: 287-298.

[53] Goda, I, Ganghoffer, JF, Czarnecki, S, et al. Optimal internal architectures of femoral bone based on relaxation by homogenization and isotropic material design. Mech Res Commun 2016; 76: 64-71.

[54] Hasanabadi, A, Baniassadi, M, Abrinia, K, et al. 3D microstructural reconstruction of heterogeneous materials from 2D cross sections: a modified phase-recovery algorithm. Comput Mater Sci 2016; 111: 107-115.

[55] Hasanabadi, A, Baniassadi, M, Abrinia, K, et al. Efficient three-phase reconstruction of heterogeneous material from 2D cross-sections via phase-recovery algorithm. J Microsc 2016; 264: 384-393.

[56] Torquato, S. Random heterogeneous materials: microstructure and macroscopic properties. Springer Science \& Business Media, 2003.

[57] Fullwood, DT, Niezgoda, SR, Adams, BL, et al. Microstructure sensitive design for performance optimization. Prog Mater Sci 2010; 55: 477-562.

[58] Fullwood, DT, Niezgoda, SR, and Kalidindi, SR. Microstructure reconstructions from 2-point statistics using phaserecovery algorithms. Acta Mater 2008; 56: 942-948.

[59] Fienup, JR. Phase retrieval algorithms: a comparison. Appl Opt 1982; 21: 2758--2769.

[60] Sheidaei, A, Baniassadi, M, Banu, M, et al. 3-D microstructure reconstruction of polymer nano-composite using FIBSEM and statistical correlation function. Compos Sci Technol 2013; 80: 47-54.

[61] Allena, R, and Cluzel, C. Heterogeneous directions of orthotropy in three-dimensional structures: finite element description based on diffusion equations. Math Mech Complex Syst 2018; 6: 339-351.

[62] Cluzel, C, and Allena, R. A general method for the determination of the local orthotropic directions of heterogeneous materials: application to bone structures using $\mu$ CT images. Math Mech Complex Syst 2018; 6: 353-367.

[63] Altenbach, H, and Eremeyev, V. On the constitutive equations of viscoelastic micropolar plates and shells of differential type. Math Mech Complex Syst 2015; 3: 273-283.

[64] Eremeyev, V, and Pietraszkiewicz, W. Material symmetry group and constitutive equations of micropolar anisotropic elastic solids. Math Mech Solids 2016; 21: 210-221.

[65] Becquart, P, Cruel, M, Hoc, T, et al. Human mesenchymal stem cell responses to hydrostatic pressure and shear stress. Eur Cells Mater 2016; 31: 160-173.

[66] Izadi, H, Baniassadi, M, Hormozzade, F, et al. Effect of 2D image resolution on 3D stochastic reconstruction and developing petrophysical trend. Transp Porous Media 2018; 125: 41-58. 